\title{
Bloqueo de rama alternante: reporte de una complicación inusual tras implante valvular aórtico transcatéter
}

\author{
Laura Betancur-Vásquez* \\ Mateo Mejía-Zuluaga** \\ Néstor Ricardo Duarte-Suarez*** \\ Tatiana María Bedoya-Jaramillo**** \\ Mauricio Duque-Ramírez ${ }^{* * * *}$
}

\begin{abstract}
* Médico Residente de Medicina Interna III año. Universidad CES. Medellín. Antioquia. Colombia. **Médico Internista. Residente de Cardiología II año. Universidad CES. Medellín. Antioquia. Colombia. ***Médico Cardiólogo. Ecocardiografista. CES Cardiología. Clínica CES. Medellín. Antioquia. Colombia. **** Médico general. CES Cardiología. Universidad CES. Medellín. Antioquia. Colombia.

*****Médico Cardiólogo. Electrofisiólogo. Jefe de postgrado de Cardiología y Electrofisiología. Universidad CES. CES Cardiología. Medellín. Antioquia. Colombia.

Correspondencia: Dr. Mauricio Duque Ramírez. Dirección: Cl. 34 \#43-66, Centro Comercial Sandiego, Torre norte, piso 11. Medellín. Antioquia. Colombia. Teléfono: +57 (4) 4447378. Correo electrónico: mauricioduquemd@gmail.com.
\end{abstract}

Resumen

Los trastornos de la conducción representan una potencial complicación de los reemplazos valvulares percutáneos. Los bloqueos alternantes de rama, aunque son inusuales, reflejan daño extenso del sistema His Purkinje con riesgo potencial de bloqueo auriculoventricular completo, por lo cual es importante su identificación y abordaje oportuno. Se presenta el caso de una paciente femenina de 82 años con múltiples comorbilidades y estenosis aórtica severa, en seguimiento médico desde hacía un año, en quien no se había realizado reemplazo quirúrgico por negativa de la paciente y por el alto riesgo intraoperatorio. Ingresó a la institución con un cuadro clínico de disnea progresiva de un mes de evolución, se realizó ecocardiograma transtorácico documentando estenosis aórtica crítica y fue llevada a implante valvular aórtico percutáneo. Por el alto riesgo de necesidad de marcapasos peri o postprocedimiento, se realizó seguimiento con monitoreo Holter, documentando bloqueo alternante de rama, el cual no progresó a bloqueo auriculoventricular completo luego de 72 horas de seguimiento, por lo que no se implantó marcapasos permanente. MÉD.UIS.2020;33(3): 37-42.

Palabras clave: Prótesis valvulares cardíacas. Trastorno del sistema de conducción. Estenosis de la válvula aórtica. Reemplazo de la válvula aórtica transcatéter.

\section{Alternating bundle branch block: report of an unusual complication after transcatheter aortic valve implantation}

\begin{abstract}
Conduction disorders represent a potential complication of percutaneous valve replacements. Alternating bundle branch block, although unusual, reflect extensive damage of the His-Purkinje system with potential risk of complete atrioventricular block, therefore, its identification and timely approach is important. We present the case of an 82 years old female patient with multiple comorbidities and a medical history of severe aortic stenosis in medical follow-up for a year, in whom surgical replacement had not been conducted due to the patient's refusal and to the high surgical risk. She was admitted to the institution referring one month of progressive shortness of breath and an echocardiogram documented critical aortic stenosis. Percutaneous aortic valve implantation was performed. Due to her high risk of needing a peri or post-procedure pacemaker, Holter monitoring was performed, documenting alternating bundle branch block, which did not progress to complete atrioventricular block after 72 hours of follow-up, in consequence, a permanent pacemaker was not implanted. MÉD.UIS.2020;33(3): 37-42.
\end{abstract}

Keywords: Heart valve prosthesis. Cardiac conduction system disease. Aortic valve stenosis. Transcatheter aortic valve replacement.

Artículo recibido el 5 de junio de 2020 y aceptado para publicación el 20 de octubre de 2020. 
¿Cómo citar este artículo?: Betancur-Vásquez L, Mejía-Zuluaga M, Duarte-Suárez NR, BedoyaJaramillo TM, Duque-Ramírez M. Bloqueo de rama alternante: reporte de una complicación inusual tras implante valvular aórtico transcatéter. MÉD.UIS.2020;33(3):37-42. doi: 10.18273/ revmed.v33n3-2020004

\section{Introducción}

La Estenosis Aórtica (EA) es la valvulopatía que más frecuentemente requiere intervención en Europa y Norte América. Su prevalencia aumenta con la edad siendo $25 \%$ a los 65 años, un $48 \%$ a los 75 años y $4-5 \%$ en menores de 65 años'. La etiología varía según la edad, pero en general $50 \%$ de los casos se deben a una calcificación degenerativa de una vávula aórtica tricúspide normal y el otro $50 \%$ a una válvula bicúspide congénita. El pilar del tratamiento de la estenosis aórtica sintomática es el reemplazo Valvular Aórtico Quirúrgico (RVAo), el cual es recomendado en pacientes con bajo riesgo quirúrgico determinado objetivamente por puntajes como STS o EuroScore $<4 \%$ y sin otros factores de riesgo como fragilidad, aorta en porcelana o secuelas de irradiación torácica. En los pacientes no candidatos a cirugía puede considerarse una prótesis aórtica por vía percutánea (TAVl: Transcatheter Aortic Valve Implantation) con tasas de sobrevida de $60-85 \%$ en pacientes de alto riesgo y hasta $95 \%$ en riesgo intermedio y una calidad de vida comparable con el reemplazo quirúrgico ${ }^{2-6}$.

En el año 2002 se implantó por primera vez una TAVI y desde entonces, la seguridad y la eficacia de este nuevo tratamiento se ha confirmado con resultados de múltiples registros multicéntricos y estudios aleatorizados ${ }^{7}$, tanto para pacientes de alto riesgo, como para moderado e incluso bajo riesgo, éste último, con varios ensayos aleatorizados en curso $^{6}$. El número de procedimientos de TAVI está creciendo rápidamente, un metanálisis estableció que anualmente 180000 pacientes en Europa, Norte América y Canadá son considerados candidatos a $\mathrm{TAVl}^{8}$. En Colombia no tenemos a la fecha grandes registros y la evidencia en el tema es por ahora anecdótica.

A pesar de ser un procedimiento menos invasivo, comparado con el RVAo, se han descrito complicaciones asociadas a TAVI tales como: complicaciones vasculares mayores, accidentes cerebro vasculares, obstrucción coronaria, infarto del miocardio, injuria renal aguda y trastornos de la conducción" ${ }^{9}$ éstos últimos con un porcentaje mayor al observado en la cirugía abierta. En cuanto a las complicaciones de la conducción y la necesidad de implantar marcapasos posterior al procedimiento debido a bloqueos AV avanzados, la TAVI ha logrado reducir su incidencia (reportes de series de casos varían la incidencia de requerimiento de implante de marcapasos entre un $6 \%$ y un $28 \%$ ). Las nuevas técnicas de implante, nuevos tipos de válvulas y una mayor experiencia del operador probablemente sean las razones por las cuales la TAVI se esté acercando al bajo riesgo que tiene la cirugía abierta ${ }^{10}$.

El bloqueo alternante de rama, como su nombre lo indica, se trata de evidenciar la presencia de ambos bloqueos de rama de forma intermitente. Este fenómeno por lo general indica la existencia de un daño extenso al sistema de conducción y el potencial riesgo de progresión a bloqueo AV completo ${ }^{11-14}$. Sobre el implante de marcapasos, se considera que es una indicación con poca evidencia científica, aunque la mayoría de la evidencia está basada en reportes de caso. Por decisión de expertos se sugiere implantar el dispositivo una vez se documente la presencia de bloqueo de rama alternante, incluso en ausencia de síncope ${ }^{15}$.

Describimos a continuación el caso de una paciente que fue llevada a TAVI (no candidata a RVAo) con alteraciones eléctricas de base dadas por Trastorno de la Conducción Auriculoventricular (AV) de primer grado y bloqueo completo de rama izquierda; motivo por el cual se realizó seguimiento clínico y con monitoreo Holter postoperatorio, documentando la presencia de bloqueo alternante de rama. Se hace necesario conocer las complicaciones asociadas a esta terapia, para llegar a un diagnóstico oportuno y acertado que impacte en los desenlaces clínicos de los pacientes.

\section{Presentación de caso}

Paciente femenina de 82 años con antecedentes de hipertensión arterial controlada, dislipidemia, prediabetes, hipotiroidismo y estenosis aórtica severa en seguimiento médico desde un año atrás, en quien no se había realizado RVAo por negativa de la paciente y por el alto riesgo quirúrgico según 
Septiembre - diciembre

la sociedad de cirujanos torácicos (STS, por sus siglas en inglés). Ingresó a la institución por cuadro clínico de un mes de evolución consistente en disnea de moderados a pequeños esfuerzos asociada a ortopnea, disnea paroxística nocturna, edema progresivo de miembros inferiores y dos episodios cercanos al síncope en las últimas dos semanas. Al ingreso hospitalario, el electrocardiograma (ECG) de 12 derivaciones evidenciaba ritmo sinusal con trastorno de la conducción AV (PR > 200 mseg) y un bloqueo completo de rama izquierda del haz de His (Ver Figura 1).

Se realizó ecocardiograma transtorácico que evidenció hipocinesia global, fracción de eyección del ventrículo izquierdo (FEVI) de 30\%, severa dilatación biauricular, alta probabilidad de hipertensión pulmonar y doble lesión aórtica con insuficiencia leve y estenosis crítica con un área valvular de $0.46 \mathrm{~cm}^{2}$ por planimetría y un gradiente medio de $30 \mathrm{mmHg}$ (Ver Figura 2).

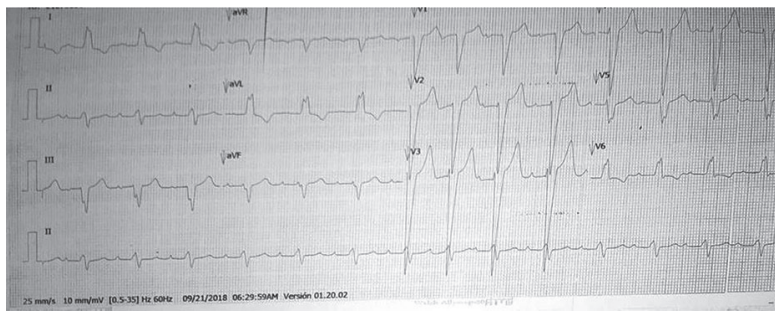

Figura 1. Electrocardiograma basal que evidencia PR prolongado y bloqueo completo de rama izquierda del Haz de His.

Fuente: autores.

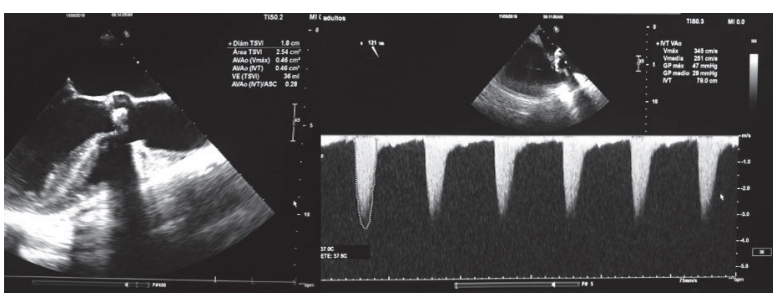

Figura 2. Estenosis valvular aórtica crítica, con área de $0.46 \mathrm{~cm} 2$, densamente calcificada, y gradientes moderadamente aumentados en relación a disfunción ventricular izquierda.

Fuente: autores.

La velocidad máxima $(395 \mathrm{~cm} / \mathrm{s})$ y los gradientes transvalvulares fueron subestimados por disfunción sistólica del ventrículo izquierdo y bajo flujo CW. No se realizó ecocardiografía dobutamina debido a la marcada reducción del área valvular, fue considerada como estenosis aórtica severa verdadera a pesar de tener bajo flujo y bajo gradiente. Se realizó angioTAC con el fin de caracterizar anatomía valvular y se evidenció estenosis aórtica calcificada severa. Dada la edad, las comorbilidades y el alto riesgo quirúrgico, se definió paciente candidata a implante valvular aórtico percutáneo.

Bajo anestesia general, monitoreo hemodinámico continuo y ecocardiografía transesofágica intraoperatoria, se avanzó por vía femoral derecha y se implantó una válvula biológica percutánea EVOLUTR de $29 \mathrm{~mm}$ autoexpandible, la cual cruzó la válvula aórtica y quedó a $7 \mathrm{~mm}$ de profundidad respecto al anillo aórtico. Posteriormente, se realizó un inflado con un balón de $23 \mathrm{~mm}$ en la prótesis implantada debido a la severa calcificación del seno posterior y aórtico derecho. Luego de la valvuloplastia, se observó una insuficiencia valvular severa, secundaria a un defecto mecánico, debido a que una de las valvas estaba fija (Ver Figura 3).

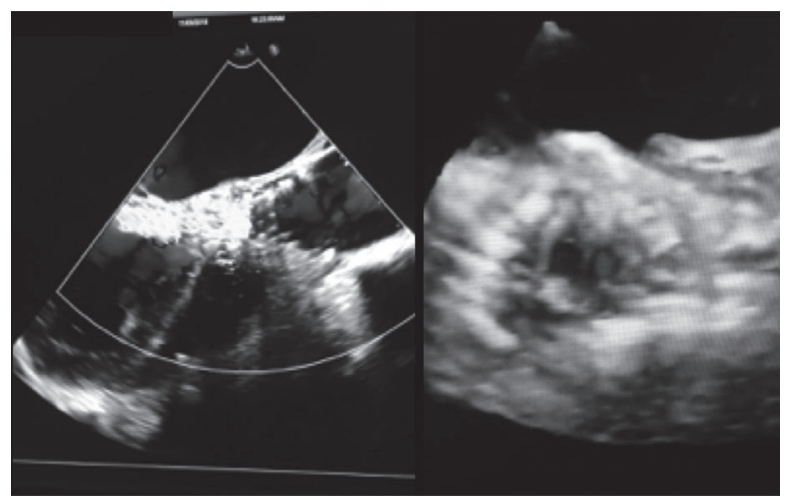

Figura 3. Prótesis valvular aórtica percutánea (TAVI), posterior a reexpansión de la prótesis para corrección de fugas paravalvulares residuales a nivel del seno de Valsalva no coronariano y a nivel del seno de Valsalva coronariano derecho, evidenciándose insuficiencia aórtica severa masiva por restricción en los movimientos a nivel de las valvas coronarias, por lo cual se decide realizar procedimiento de valve in valve (válvula en válvula).

Fuente: autores.

Por esta razón, se avanzó una segunda válvula de las mismas características (EVOLUTR-29 mm) que sellevó a través de la válvula previamente colocada y se liberó 1 a $2 \mathrm{~mm}$ por debajo de la válvula inicial, con la cual se corrigió completamente el problema presentado. Finalmente, se observó un resultado favorable con un gradiente transvalvular máximo de $5 \mathrm{mmHg}$ y 2 fugas paravalvulares leves que desaparecieron en el seguimiento. Durante la intervención no se presentó inestabilidad hemodinámica ni alteraciones del ritmo.

Fue trasladada a cuidados intensivos con marcapasos transitorio profiláctico, el cual se retiró a las 72 
horas previo al traslado a habitación convencional. La ecocardiografía transtorácica de control, realizada a los nueve días de la intervención, previo al alta hospitalaria, evidenció hipocinesia global, movimiento asincrónico del septo interventricular, prótesis valvular en posición aórtica (válvula en válvula) con gradientes normales y fuga con insuficiencia leve (Ver Figura 4).

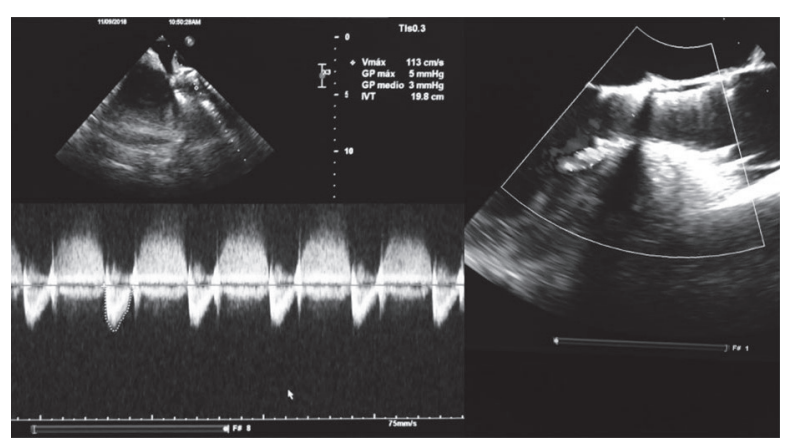

Figura 4. Posterior al implante de válvula en válvula, se encuentra la válvula normoposicionada, con gradientes normales y una insuficiencia paravalvular residual leve.

Fuente: autores.

Previo al alta, se realizó monitoreo Holter de 24 horas en el cual se documentó la presencia de un bloqueo alternante de rama (Ver Figura 5).

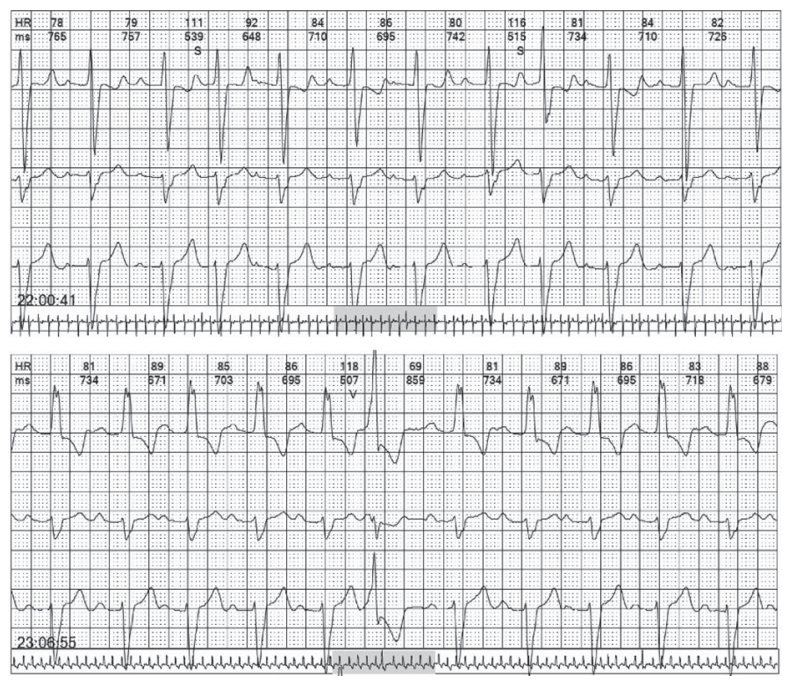

Figura 5. Monitoreo Holter de 24 horas que demuestra bloqueo alternante de rama. Se observan diferentes morfologías y polaridades de QRS, con una hora de diferencia.

Fuente: autores.

Luego de una decisión conjunta entre cardiología, hemodinámica, electrofisiología y la paciente, se optó por el seguimiento clínico para determinar la necesidad futura de un marcapasos. La paciente tuvo una evolución sin complicaciones y fue dada de alta en buenas condiciones; dos años luego de la intervención se encontraba asintomática, con una FEVI recuperada hasta $8 \%$ y en los dos monitoreos Holter a los que fue sometida, no se evidenció bloqueo alternante ni otras complicaciones eléctricas.

\section{Discusión}

Conociendo entonces la importancia y epidemiología de la estenosis aórtica y los resultados obtenidos por la TAVI frente a pacientes con riesgo intermedio ${ }^{5}$ probablemente estemos ante una tecnología que cada vez será más frecuente observar en la práctica clínica y como toda intervención novedosa, debemos conocer las potenciales complicaciones que la acompañan.

No hay duda de que la TAVI es una intervención menos invasiva que la cirugía, sin embargo, existen potenciales complicaciones que deben ser conocidas, entre ellas se encuentran la obstrucción coronaria, la lesión de la válvula mitral, fugas paravalvulares, ruptura del anillo, taponamiento cardíaco y bloqueos $A V^{16}$. Un metaanálisis en el que se incluyeron 4 estudios con 3806 pacientes en total, comparó TAVI con reemplazo quirúrgico de válvula aórtica, demostrando significativamente menor riesgo de lesión renal (HR 0.61 IC95\% 0.41-0.9), menor riesgo de nueva fibrilación atrial (HR 0.46 IC95\% 0.34-0.63), menor riesgo de sangrado mayor (HR 0.57 IC $95 \% 0.35$ 0.92), mayor riesgo de complicaciones vasculares mayores (HR 2.46 IC95\% 1.49-4.05) y necesidad de implante de marcapasos (HR 2.12 IC $95 \%$ 1.13-3.99) ${ }^{17}$.

Con respecto a las complicaciones eléctricas, los bloqueos AV completos posteriores a TAVI se presentan presumiblemente como consecuencia de la presión aplicada en el tejido de conducción, localizado a nivel subendocárdico en el tracto de salida del ventrículo izquierdo y en el septum interventricular causando una alteración transitoria o permanente del tejido de conducción a este nivel ${ }^{16}$. Esto se refuerza en el hecho de que aquellas prótesis que se extienden hacia el ventrículo se asocian con mayor incidencia de anormalidades de la conducción, especialmente con la aparición de un nuevo bloqueo de rama izquierda. Los factores de riesgo para la ocurrencia de este fenómeno son la sobredistensión agresiva y las anormalidades preexistentes de la conducción distal9,18,19. 
Para las complicaciones eléctricas, se han logrado identificar ciertos factores de riesgo como son: el grosor de la pared septal, el grosor de la cúspide no coronariana, bloqueo de rama preexistente, la profundidad de implante en el tracto de salida del ventrículo izquierdo, la expansión post implante de la prótesis (sobreexpansión) y el tipo de prótesis ${ }^{20-23}$.

El bloqueo alternante de rama se presenta como una manifestación de lesión extensa del sistema His-Purkinje con riesgo potencial de bloqueo AV completo; ; sin embargo, la evidencia de esta complicación en el contexto de TAVI es escasa. Un estudio retrospectivo holandés en el que se incluyeron 155 pacientes de $80 \pm 7$ años con bloqueo de rama izquierda posterior a $\mathrm{TAVI}^{24}$, reportó que de los pacientes que requerían marcapasos luego de la intervención (24\%), un $46 \%$ presentaban bloqueo AV completo, $41 \%$ bloqueo AV intermitente, un $5 \%$ bloqueo AV de primer o segundo grado y $5 \%$ bloqueo alternante de rama ${ }^{25}$. En un estudio canadiense con pacientes llevados a TAVI, se requirió implante de marcapasos en $15.6 \%$ de pacientes, de los cuales el $3.8 \%$ tenían bloqueo alternante de rama. Resultados similares han sido documentados en otros estudios ${ }^{26}$. Se ha demostrado que durante el postoperatorio temprano, las alteraciones eléctricas pueden ser más frecuentes y no todas acarrean un mal pronóstico a largo plazo e incluso en un estudio realizado con la válvula SAPIEN 3, se observó que un porcentaje de estas alteraciones se estabilizan o desaparecen en el seguimiento a 12 meses, demostrando la importancia de individualizar el riesgo de cada paciente para definir si es candidato a implante de marcapasos o a una estrategia más conservadora, como lo es el seguimiento clínico ${ }^{27,28}$.

Aunque por el momento no se tenga evidencia científica sólida que genere recomendaciones fuertes, algunas revistas, como JACC y Current Cardiology Reviews, han publicado un panel de expertos sobre como actuar frente a las diferentes alteraciones y complicaciones eléctricas que se pueden encontrar posterior a una $\mathrm{TAVI}^{27,29}$ y qué conductas tomar una vez se identifiquen. Sin embargo, no se habla específicamente del bloqueo alternante de rama, de aquí la importancia que éste sea reconocido como una potencial complicación de la TAVI y por esto la relevancia de comenzar a crear evidencia alrededor de las complicaciones y sus desenlaces.

\section{Conclusiones}

A pesar de que la TAVI se constituye como una intervención menos invasiva que el RVAo y es una opción para pacientes no candidatos a reemplazo quirúrgico de válvula aórtica, cuenta con complicaciones y riesgos que deben conocerse, entre ellos están las alteraciones eléctricas, como el bloqueo alternante de rama presentado en el artículo, el cual acarrea un potencial riesgo de bloqueo AV completo. El bloqueo alternante de rama evidenciado en la paciente puede ser explicado probablemente por la necesidad del implante de válvula en válvula que pudo llevar a una sobredistensión excesiva del tejido endocárdico comprometiendo transitoriamente un sistema de conducción previamente enfermo. Dado que las alteraciones eléctricas asociadas a TAVI pueden ser transitorias, el seguimiento clínico estrecho puede ser considerado como una conducta razonable.

\section{Financiación}

Ninguna.

\section{Conflicto de intereses}

Se declara que no existe ningún conflicto de interés.

\section{Referencias bibliográficas}

1. Otto CM, Lind BK, Kitzman DW, Gersh BJ, Siscovick DS. Association of aortic-valve sclerosis with cardiovascular mortality and morbidity in the elderly. N Engl J Med. 1999; 341(3):142-147.

2. Baumgartner H, Falk V, Bax JJ, De Bonis M, Hamm C, Holm PJ, et al. 2017 ESC/EACTS Guidelines for the management of valvular heart disease. Eur Heart J. 2017;38(36):2739-91.

3. Paredes A, Martínez A. Prótesis valvular aórtica percutánea: ¿Qué debemos saber? Rev Chil Cardiol. 2012;31(1):55-62.

4. Blier L, Sarrazin JF, Thibault B, Rivard L, Gula L, Sit PL, et al. Transcatheter versus Surgical Aortic-Valve Replacement in HighRisk Patients. N Engl J Med. 2011;364(23): 2187-98.

5. Leon MB, Smith CR, Mack MJ, Makkar RR, Svensson LG, Kodali SK, et al. Transcatheter or surgical aortic-valve replacement in intermediate-risk patients. N Engl J Med. 2016;374(17):1609-20.

6. Thyregod HGH, Steinbrüchel DA, Ihlemann N, Nissen H, Kjeldsen BJ, Petursson P, et al. Transcatheter versus surgical aortic valve replacement in patients with severe aortic valve stenosis: 1 -year results from the all-comers NOTION randomized clinical trial. J Am Coll Cardiol. 2015;65(20):2184-94.

7. Cribier A, Eltchaninoff H, Bash A, Borenstein N, Tron C, Bauer F, et al. Percutaneous transcatheter implantation of an aortic valve prosthesis for calcific aortic stenosis: First human case description. Circulation. 2002;106(24):3006-8.

8. Durko AP, Osnabrugge RL, Van Mieghem NM, Milojevic M, Mylotte D, Nkomo VT, et al. Annual number of candidates for 
transcatheter aortic valve implantation per country: current estimates and future projections. Eur Heart J. 2018;39(28):263542.

9. Leon MB, Webb JG, Kodali S, Tuzcu ME, Kapadia S, Masson JB, et al. Transcatheter Aortic Valve Implantation: Review of the Nature, Management, and Avoidance of Procedural Complications. JCIN [Internet]. 2009;2(9):811-20. Disponible en: http://dx.doi. org/10.1016/j.jcin.2009.07.005

10. Karyofillis P, Kostopoulou A, Thomopoulou S, Habibi M, Livanis E, Karavolias G, et al. Conduction abnormalities after transcatheter aortic valve implantation. J Geriatr Cardiol. 2018;15(1):105-12.

11. Fazzini PF, Marchi F, Pucci P. Prognostic significance of the intraventricular blocks during acute myocardial infarction. G Ital Cardiol. 1975;5(4):526-35.

12. Beck OA, Hochrein H. Alternating and intermittent bilateral bundle-branch block in acute myocardial infarct with development of total atrioventricular block. Z Kardiol. 1976 Mar;65(3):245-55.

13. Ogura Y, Kato J, Ogawa Y, Shiokoshi T, Kitaoka T, Suzuki T, et al. A case of alternating bundle branch block in combination with intra-Hisian block. Int Heart J. 2005 Jul;46(4):737-44.

14. Fisch C, Zipes DP, McHenry PL. Rate dependent aberrancy. Circulation. 1973 Oct;48(4):714-24.

15. Brignole M, Auricchio A, Baron-Esquivias G, Bordachar P, Boriani G, Breithardt O-A, et al. 2013 ESC guidelines on cardiac pacing and cardiac resynchronization therapy: the Task force on cardiac pacing and resynchronization therapy of the European Society of Cardiology (ESC). Developed in collaboration with the European Heart Rhythm Association (EHRA). Europace. 2013 Aug;15(8):1070-118.

16. Baumgartner H, Falk V, Bax JJ, De Bonis M, Hamm C, Holm PJ, et al. 2017 ESC / EACTS Guidelines for the management of valvular heart disease. Eur Heart J. 2017;38(36): 2739-91.

17. Siontis GCM, Praz F, Pilgrim T, Mavridis D, Verma S, Salanti G, et al. Transcatheter aortic valve implantation vs. surgical aortic valve replacement for treatment of severe aortic stenosis: A metaanalysis of randomized trials. Eur Heart J. 2016;37(47):35033512 .

18. Clayton B, Morgan-Hughes G, Roobottom C. Transcatheter aortic valve insertion (TAVI): a review. Br J Radiol. 2014; 87(1033): 20130595.

19. Arora A, Misenheimer JA, Ramaraj R. Transcatheter Aortic Valve Replacement: Comprehensive Review and Present Status. Tex Heart Inst J. 2017;44(1):29-38.

20. Piazza N, Nuis R-J, Tzikas A, Otten A, Onuma Y, García-García $\mathrm{H}$, et al. Persistent conduction abnormalities and requirements for pacemaking six months after transcatheter aortic valve implantation. EuroIntervention. 2010 Sep;6(4):475-84.

21. Baan JJ, Yong ZY, Koch KT, Henriques JPS, Bouma BJ, Vis MM, et al. Factors associated with cardiac conduction disorders and permanent pacemaker implantation after percutaneous aortic valve implantation with the CoreValve prosthesis. Am Heart J. 2010;159(3):497-503.

22. Roten L, Wenaweser P, Delacrétaz E, Hellige G, Stortecky S, Tanner $\mathrm{H}$, et al. Incidence and predictors of atrioventricular conduction impairment after transcatheter aortic valve implantation. Am J Cardiol. 2010;106(10):1473-80.

23. Erkapic D, Kim WK, Weber M, Möllmann H, Berkowitsch A, Zaltsberg S, et al. Electrocardiographic and further predictors for permanent pacemaker requirement after transcatheter aortic valve implantation. Europace. 2010;12(8):1188-90.

24. Ozier D, Zivkovic N, Elbaz-Greener G, Singh SM, Wijeysundera HC. Timing of Conduction Abnormalities Leading to Permanent Pacemaker Insertion After Transcatheter Aortic Valve Implantation-A Single-Centre Review. Can J Cardiol [Internet]. 2017;33(12):1660-7. Disponible en: http://dx.doi.org/10.1016/j. cjca.2017.08.012

25. Nijenhuis VJ, Van Dijk VF, Chaldoupi SM, Balt JC, Ten Berg JM. Severe conduction defects requiring permanent pacemaker implantation in patients with a new-onset left bundle branch block after transcatheter aortic valve implantation. Europace [Internet]. 2017;19(6):1015-21. Disponible en: https://academic. oup.com/europace/article-lookup/doi/10.1093/europace/euw174

26. Becker M, Blangy H, Folliguet T, Villemin T, Freysz L, Luc A, et al. Analyse rétrospective de l'incidence, des indications et des facteurs prédictifs d'implantation de stimulateurs cardiaques définitifs après remplacement valvulaire aortique percutané. Arch Cardiovasc Dis [Internet]. 2017;110(10):508-16. Disponible en: http://dx.doi.org/10.1016/j.acvd.2017.03.004

27. Rodés-Cabau J, Ellenbogen KA, Krahn AD, Latib A, Mack M, Mittal $\mathrm{S}$, et al. Management of Conduction Disturbances Associated With Transcatheter Aortic Valve Replacement: JACC Scientific Expert Panel. J Am Coll Cardiol [Internet]. 2019;74(8):1086-106. Disponible en: https://doi.org/10.1016/j.jacc.2019.07.014

28. Dolci G, Vollema EM, van der Kley F, de Weger A, Marsan NA, Delgado V, et al. One-Year Follow-Up of Conduction Abnormalities After Transcatheter Aortic Valve Implantation With the SAPIEN 3 Valve. Am J Cardiol. 2019;124(8):1239-45.

29. Russo E, Potenza DR, Casella M, Massaro R, Russo G, Braccio M, et al. Rate and Predictors of Permanent Pacemaker Implantation After Transcatheter Aortic Valve Implantation: Current Status. Curr Cardiol Rev. 2019;15(3):205-18.[JV1] 